\title{
Fréquence de quelques chiroptères durant l'Holocène
}

\author{
Michel Blant, La Chaux-de-Fonds, Marco Moretti, \\ Bellinzone, Willy Tinner, Berne et Zurich
}

\section{Introduction}

La connaissance des peuplements de chiroptères était jusqu'à présent confinée dans une dimension plus spatiale que temporelle. Or, ces mammifères sont actuellement considérés comme de précieux indicateurs de l'état environnemental, et pour la plupart menacés au nord ou au sud des Alpes suisses (Duell 1994; MoRetTI et al. 2003) comme au niveau mondial (INTERnational Union for Conservation of NatUre and Natural Resources (IUCN) 2000).

La présente étude basée sur des datations radiocarbones d'ossements subfossiles issus de grottes avait pour objet de tenter de relier la présence de quelques traceurs choisis à des facteurs environnementaux tels que les successions végétales et le climat durant l'Holocène. Cette approche cherche à vérifier les hypothèses émises dans la littérature concernant la composition de ce peuplement faunique en rapport avec l'évolution de la forêt (avancée, composition des peuplements, structure) et du paysage (déboisements dès le Néolithique, incendies de forêts). Des collectes de matériel importantes (Blant et al. 2004; Morel \& Trüssel 1997) ont été réalisées par les spéléologues dans plusieurs cantons du sud (Tessin) et du nord des Alpes (Obwald, Berne, Fribourg). Comme ces gisements n'avaient jusqu'ici pas été valorisés du point de vue chronologique et paléoécologique, un projet d'étude a été lancé en 2006 par l'Institut suisse de spéléologie et de karstologie (ISSKA). Les données obtenues ont été analysées en collaboration avec le Groupe de recherche sur les écosystèmes insubriens de l'institut fédéral de recherches sur la forêt, la neige et le paysage (WSL) et la Section de paléoécologie de l'Université de Berne.

\section{Matériel et méthodes}

Le matériel analysé a été récolté bénévolement par les spéléologues de la Société suisse de spéléologie (section du Tessin (SSS-TI), section de Berne (SGH-Bern), Naturerbe Karst und Höhlen Obwalden (NeKO), Spéléo-club des Préalpes fribourgeoises (FriBAT/ SCPF)) dans les gisements localisés sur la carte de la figure 1 . Les crânes datés ont été collectés à même le sol ou parfois dans des couches minces de sédiments. Ils proviennent d'animaux morts durant leur léthargie hivernale.
Un noyau d'espèces de chiroptères à écologie sylvicole a été choisi, potentiellement sensible aux variations climatiques. Des résultats partiels relatifs à trois traceurs, le murin de Bechstein (Myotis bechsteinii), le petit murin (Myotis blythii) et le murin de Brandt (Myotis brandtii) sont présentés ici. Le premier peut être qualifié de mésophile (répartition médio-européenne), le second de franchement thermophile (répartition méditerranéenne) et le troisième de psychrophile (répartition boréo-alpine). Ces trois espèces sont rares, mais encore actuelles en Suisse.

Les datations radiocarbones AMS (accelerated mass spectrometry) ont été effectuées par le Dr Georges Bonani de l'Institut de physique des particules de l'École Polytechnique Fédérale de Zurich (EPFZ).

Le proxy utilisé en référence pour une comparaison des données obtenues à la température moyenne durant l'Holocène est la courbe de la température estivale (mois de juillet) de l'air reconstituée par HeIRI et al. (2003) sur la base de la présence de peuplements de chironomidés dans un lac subalpin suisse (Hinterburgsee, Préalpes bernoises, $1515 \mathrm{~m}$ ).

\section{Résultats}

Au total, 31 datations ont été effectuées sur ces trois espèces $(M$. bechsteinii: $\mathrm{n}=14 ; M$. blythii: $\mathrm{n}=4 ; M$. brandtii: $\mathrm{n}=13$ ). Elles sont situées chronologiquement sur la figure 2 .

Les plus anciennes dates obtenues pour chacune des trois espèces proviennent d'échantillonnages effectués au Monte Generoso (Grotta del Canalone) dans les Préalpes tessinoises (M. bechsteinii: 6255-6007 cal BC M. blythii: $8740-8340$ cal BC; $M$. brandtii: $6268-6020 \mathrm{cal}$ $\mathrm{BC})$. Les plus récentes proviennent d'une grotte du massif des Sieben Hengste (D 7.1) dans les Préalpes bernoises ( $M$. bechsteinii: 427-618 cal AD) et d'une grotte du massif de la Melchsee-Frutt (Bettenhöhle) dans le canton d'Obwald (M. brandtii: 430-660 cal AD).

\section{Discussion}

\subsection{Végétation}

Les données obtenues pour $M$. bechsteinii couvrent un intervalle de temps allant de l'Atlantique ancien au Subatlantique. Elles s'inscrivent dans la succession forestière holocène, depuis la fin des forêts de pins 


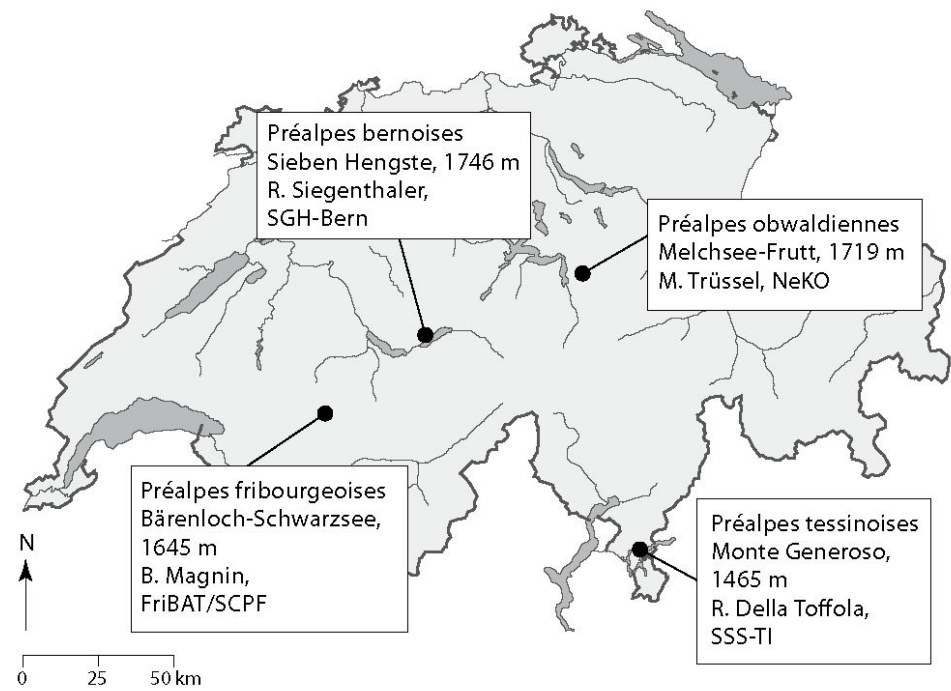

Fig. 1: Situation des grottes

Lage der Höhlen

Location of the caves

Dessin: M. BLANT; cartographie: L. BaUmanN

(Pinus sylvestris, P.cembra) et bouleaux (Betula) à l'apparition des forêts mixtes de chênes (Quercus, Corylus avellana), puis de sapins (Abies alba), de hêtres (Fagus sylvatica) et d'épiceas (Picea abies) (HeIri et al. 2003; Tinner et al. 1999; Tinner et al. 2005) (fig. 2). L'espacement des dates obtenues à partir de $3000 \mathrm{cal} \mathrm{BC}$ permet d'émettre l'hypothèse d'un fléchissement des populations de $M$. bechsteinii amorcé depuis le début du Subboréal. Pour cette espèce des forêts caducifoliées (Bangoe 2001; Meschede \& Heller 2003), le glissement de la chênaie vers la hêtraie pourrait représenter une péjoration de son habitat (diminution des gîtes ou des ressources alimentaires?), qui s'accélère ensuite avec les défrichements d'origine anthropique.

Dans les sondages, $M$. blythii apparaît déjà au Préboréal (fig. 2), soit à l'apogée des forêts de pins et d'arolles (Pinus sylvestris, P. cembra), avec le déclin du bouleau (Betula) et l'apparition de l'orme (Ulmus) et du chêne (Quercus) dans les espèces dominantes au sud des Alpes (Tinner et al. 1999). Sa présence se poursuit dans les phases du Boréal et de l'Atlantique, jusqu'au début du Néolithique. A ce moment, outre le chêne, les forêts sont dominées par le noisetier (Corylus avellana) et le sapin (Abies alba) (fig. 2). Cette espèce chasse dans les habitats steppiques chauds les orthoptères et autres proies des prairies (ARLETTAZ 1995). Sa présence à une époque précoce de l'Holocène doit être reliée à la disponibilité en habitats semi-ouverts, à l'image de la structuration des pinèdes actuelles, en l'absence de milieux ouverts d'origine anthropique.
Les dates obtenues pour $M$. brandtii se concentrent sur deux périodes, la première s'étendant de l'Atlantique jusqu'au début du Subboréal, la seconde dans le Subatlantique. Cette espèce a pu fréquenter les forêts montagnardes, conformément à son écologie d'espèce des forêts boréales au sein de sa distribution nord-européenne (STRELKov 1983), comme celles de plaine, vu sa présence actuelle dans des forêts mixtes en Europe centrale (Woloszyn 1970). Elle s'est adaptée sans doute dès le Néolithique aux paysages semiouverts dans lesquels on la rencontre maintenant en Allemagne (Meschede \& Heller 2003).

\subsection{Climat}

On constate une agrégation de données concernant M. bechsteinii principalement durant l'optimum climatique de l'Holocène (fig. 2). Ces résultats confirment qu'à cette période l'espèce a bénéficié de conditions écologiques optimales aboutissant à son apogée en Europe centrale, hypothèse formulée sur la base de sa forte représentation dans la documentation subfossile holocène (nombreux auteurs in BAAGOE 2001). Cette espèce actuellement rare en Suisse devait donc y être plus abondante comme le suggèrent MoREL \& TRÜsSEL (1997). Une concentration de données obtenue entre 6800 et $6200 \mathrm{cal} \mathrm{BP}$ correspond d'ailleurs à une longue phase climatique particulièrement chaude (et probablement sèche) située entre deux phases plus froides (CE-4, 7500-7100 cal BP et CE5, 6100-5650 cal $\mathrm{BP})$ correspondant à la température actuelle (HAAs et al. 1998). 


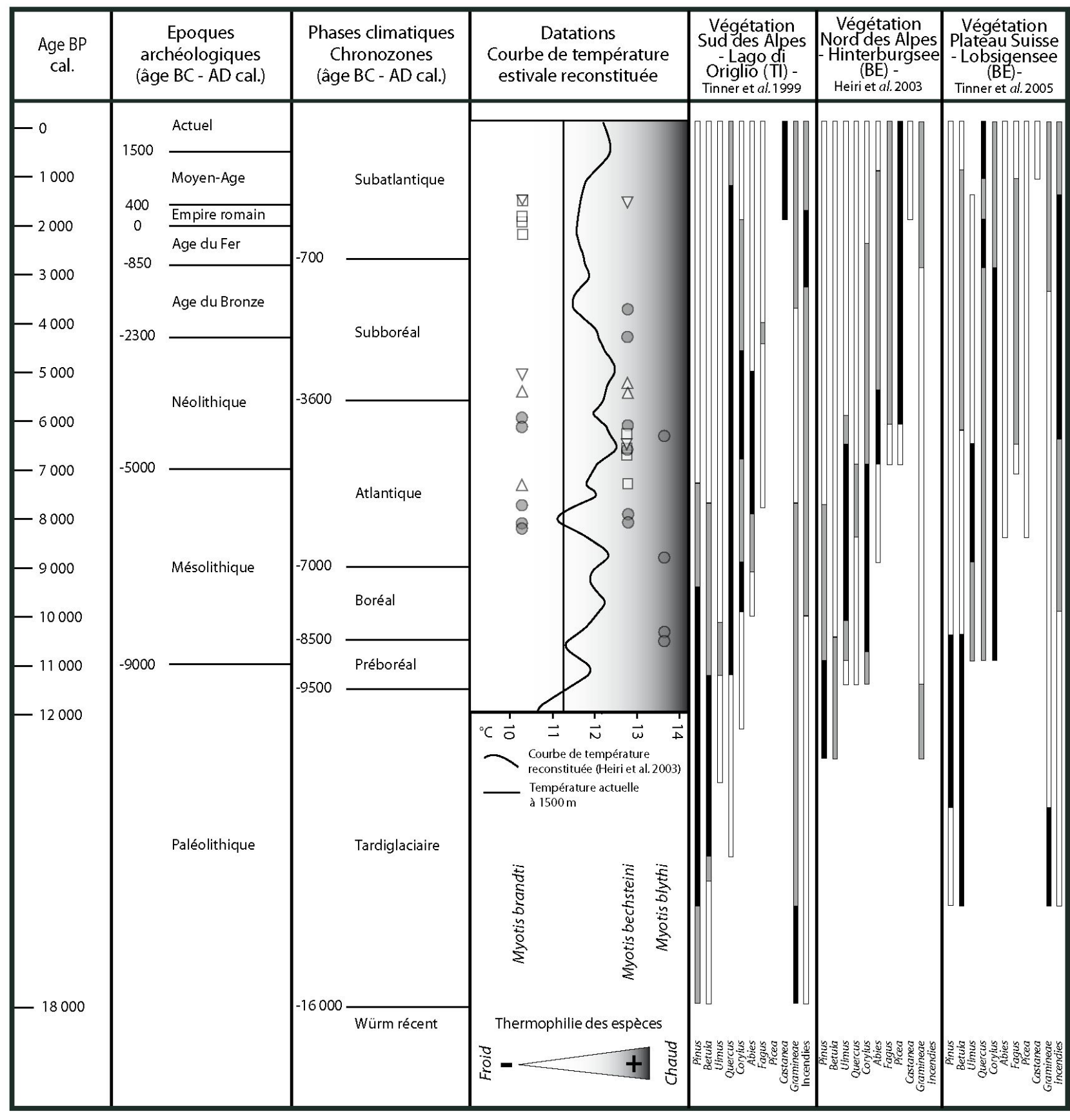

\section{Grottes}

$\triangle$ Bärenloch (FR)

$\nabla$ Sieben Hengste (BE)

$\square \quad$ Melchsee-Frutt (OW)

- Grotta del Canalone (TI) $--\overline{\text { sud des alpes }}$

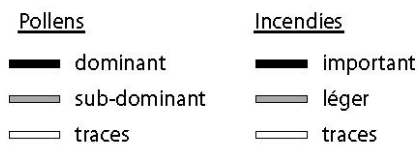

Fig. 2: Chronologie des datations et de la végétation Chronologie der Altersbestimmungen und der Flora Chronology of the dating and the vegetation

Source: Température d'après Heiri et al. 2003, végétation d'après Tinner et al. 1999, Heiri et al. 2003 et Tinner et al. 2005; dessin: M. BLANT 
La première donnée concernant $M$. blythii apparaît à la fin du Préboréal, lorsque la température a atteint la moyenne actuelle. Le nombre réduit de données ne permet aucune interprétation.

Pour M. brandtii, les données sont dispersées sans rapport apparent à la température.

\subsection{Chronologie}

Si les données présentées ici n'apportent guère de faits nouveaux concernant $M$. bechsteinii, les dates absolues permettent au moins de confirmer les hypothèses avancées dans la littérature et basées sur sa fréquence dans des thanatocénoses souvent non datées. Ces dates sont par ailleurs notablement plus précoces dans l'Holocène que celles obtenues par BAUER (1987) situées de $2500 \mathrm{BC}$ à $400 \mathrm{AD}$ environ. BAAGOE (2001) signale sa présence dans une thanatocénose de la fin du Préboréal, mais la date absolue (9008-8472 cal BC) est obtenue en fait sur un Myotis dasycneme. Les données concernant $M$. blythii sont par contre nouvelles puisqu'elles authentifient sa présence dans une phase précoce de l'Holocène. Les données fragmentaires concernant cette espèce situent son retour en Hongrie et en Autriche aux environs de 7000 BP seulement (Topál \& Ruedi 2001). La fréquence holocène de $M$. brandtii est mal documentée. Les données reportées ici pour la Suisse sont donc originales pour cette espèce.

\subsection{Critique de la méthode}

Le choix des pièces à dater comporte indéniablement un aspect aléatoire. Les hypothèses formulées dans ce travail relèvent cependant d'un nombre de datations aussi élevé que possible compte tenu des coûts et moyens à disposition. Ces premières tentatives d'interprétation méritent cependant encore d'être croisées avec des résultats issus d'un plus grand nombre d'espèces et de datations.

\section{Conclusions}

Les résultats suggèrent une corrélation entre la présence de certaines espèces de chiroptères et le climat de l'Holocène. Au niveau de la végétation, la structure des peuplements et les modifications d'origine anthropique pourraient aussi jouer un rôle quant à leur abondance à certaines phases. Les datations obtenues sur quelques specimens de Myotis bechsteinii et $M$. blythit sont plus précoces que celles reportées dans la littérature pour ces espèces au début de l'Holocène.

\section{Remerciements}

Les datations et l'analyse des résultats ont été soutenues financièrement par le Musée cantonal d'histoire naturelle du canton du Tessin (MCSN, Lugano), la section bernoise de la Société suisse de spéléologie (SGH-Bern), l'Office fédéral de l'environnement (OFEV), l'Institut fédéral de recherches sur la forêt, la neige et le paysage (WSL) (Groupe de recherche sur les écosystèmes insubriens, Unité écotones, Bellinzone) et l'Université de Berne (Institut des sciences botaniques, Section de paléoécologie).

\section{Bibliographie}

Arlettaz, R. (1995): Ecology of the sibling mouseeared bats (Myotis Myotis and Myotis blythii). Zoogeography, niche, competition, and foraging. - Martigny: Horus Publishers.

BaAgoe, H.J. (2001): Myotis bechsteinii - Bechsteinfledermaus. - In: Niethammer, J. \& F. KRAPP (Hrsg.) Handbuch der Säugetiere Europas. Band 4: Fledertiere. - Wiebelsheim: Aula-Verlag: 443-472.

BaUER, K. (1987): Die holozäne Fledermausfauna des Katerloches bei Weiz, Steiermark (Mammalia, Chiroptera). - In: Mitteilungen der Abteilung Zoologie des Landesmuseums Joanneum 40: 25-40.

Blant, M., Moretti, M., Della Toffola, R. \& R. Pierallini (2004): La fauna olocenica del Sud delle Alpi svizzere. Chirotteri e Mammiferi terrestri tra passato e presente (Grotta del Canalone, Monte Generoso, Ticino). - In: Bollettino della Società Ticinese di Scienze Naturali 92, 1-2: 31-44.

Duelli, P. (1994): Listes rouges des espèces animales menacées de Suisse. - Berne: Office fédéral de l'environnement et du paysage.

HaAs, J.N., Richoz, I., Tinner, W. \& L. Wick (1998): Synchronous Holocene climatic oscillations recorded on the Swiss Plateau and at timberline in the Alps. - In: The Holocene 8: 301-309.

Heiri, O., Wick, L., VAN Leeuwen, J.F.N., VAN der KnaAP, W.O. \& A.F. LOTTER (2003): Holocene tree immigration and the chironomid fauna of a small Swiss subalpine lake (Hinterburgsee, $1515 \mathrm{~m}$ asl). - In: Palaeogeography, Palaeoclimatology, Palaeoecology 189: 35-53.

INTERnational UNion fOR CONSERVATION OF NATURE AND Natural Resources (IUCN) (2000): The 2000 IUCN red list of threatened species. - Gland: IUCN.

Meschede, A. \& K.-G. Heller (2003): Ecologie et protection des chauves-souris en milieu forestier. - = Le Rhinolophe 16, Genève: Muséum d'histoire naturelle de la Ville de Genève.

Morel, P. \& M. Trüssel (1997): Datierter Nachweis eines holozänen Skelettes einer Wimperfledermaus (Myotis emarginatus) aus einer Höhle der Obwaldner Voralpen, Melchsee-Frutt, Kerns OW. - In: Mitteilungen der Naturforschenden Gesellschaft Ob- und Nidwalden 1:106-115.

Moretti, M., Roesli, M., Gamboni, A.-S. \& T. MaDDalena (2003): I pipistrelli del Cantone Ticino. - = Memorie della Società Ticinese di Scienze Naturali 6, Lugano: Società Ticinese di Scienze Naturali. 
STRelkov, P.P. (1983): Myotis mystacinus and Myotis brandti in the USSR and interrelations on these species. Part 2. - In: Zoologicheskii zhurnal 62: 259-270.

Tinner, W., Hubschmid, P., Wehrli, M., Ammann, B. \& M. CONEDERA (1999): Long-term forest-fire ecology and dynamics in southern Switzerland. - In: Journal of Ecology 87: 273-289.

Tinner, W., Conedera, M., Ammann, B. \& A.F. Lotter (2005): Fire ecology north and south of the Alps since the last ice age. - In: The Holocene 15, 8: 1214-1226.

TopáL, G. \& M. RuedI (2001): Myotis blythii - Kleines Mausohr. - In: Niethammer, J. \& F. KRAPP (Hrsg.): Handbuch der Säugetiere Europas. Band 4: Fledertiere. - Wiebelsheim: Aula-Verlag: 209-255.

Woloscyn, B.W. (1970): Holoceńska fauna nietoperzy (Chiroptera) z jaskiń tatrzańskich. The Holocene chiropteran fauna from the Tatra Cave. $-=$ Folia quaternaria 35, Kraków: Polska Akademia Nauk - Oddział w Krakowie.

\section{Résumé: Fréquence de quelques chiroptères durant l'Holocène}

Des datations au ${ }^{14} \mathrm{C}$ de crânes de chauves-souris ont été effectuées sur des collectes provenant de grottes du sud et du nord des Alpes suisses. Le but de ce projet était de cerner les époques d'apparition de quelques espèces forestières considérées comme traceurs de périodes chaudes ou froides, puis de mettre en relation leur insertion chronologique avec les successions végétales de l'Holocène. Les données montrent une présence de longue durée pour Myotis bechsteinii et Myotis brandtii, un traceur mésothermophile et un traceur psychrophile. Une espèce thermophile méditerranéenne, Myotis blythii, apparaît dans un intervalle de temps plus restreint. La fréquence des espèces peut être expliquée au moins en partie par les successions végétales et les déboisements. En outre, les résultats suggèrent une corrélation entre la présence d'espèces rares thermophiles comme Myotis bechsteinii et des phases à climat chaud pendant l'Holocène.

Mots-clés: chauves-souris, datation, forêt, climat, chronologie

\section{Summary: Occurrence of bat species during the Holocene}

One aim of the project described herein was to identify the temporal occurrence of several forest-dwelling bat species considered markers of warm and cold periods and to investigate possible relations to vegetational development during the Holocene. For this purpose, bat skulls were collected in caves in southern and northern Switzerland and ${ }^{14} \mathrm{C}$ dated. The results show that species, such as Myotis bechsteinii (mesothermophile) and Myotis brandtii (psychrophile), occupied these areas during a long period, whereas occurrence of Myotis blythii (a thermophile Mediterranean species) is restricted to a relatively short time period. The abundance of the species observed can be at least partially explained by vegetational development and deforestation. Moreover, the results suggest a link between the presence of rare mesothermophile species, such as Myotis bechsteinii, and warm Holocene climatic conditions.

Keywords: bats, dating, forest, climate, chronology

\section{Zusammenfassung: Vorkommen einiger Fledermaus- arten während des Holozäns}

Fledermausschädel aus Höhlen der Schweizer Nordund Südalpen wurden karbondatiert. Ein Ziel dieses Projektes war, das zeitliche Vorkommen einiger waldbewohnender Arten, die als wärme- oder kälteliebend gelten, zu bestimmen und ihr Verhältnis zur holozänen Vegetationsentwicklung zu untersuchen. Die Resultate zeigen eine langandauernde Anwesenheit von Myotis bechsteinii und Myotis brandtii, die als wärme- bzw. kälteliebend gelten. Eine thermophile Mittelmeerart, Myotis blythii, erscheint in einem kürzeren Zeitintervall. Das Vorkommen der Arten kann, zumindest teilweise, durch die Vegetationsentwicklung und die Entwaldung erklärt werden. Die Daten weisen auf einen Zusammenhang zwischen der Anwesenheit seltener, mesothermophiler Arten wie Myotis bechsteinii und warmen klimatischen Verhältnissen während des Holozäns hin.

Schlüsselwörter: Fledermäuse, Altersbestimmung, Wald, Klima, Chronologie

Dr. Michel Blant, Institut suisse de spéléologie et de karstologie (ISSKA), Case postale 818, CH-2301 La Chauxde-Fonds, en collaboration avec le Musée cantonal d'histoire naturelle du Tessin, CH-6900 Lugano, Suisse.

e-mail: michel.blant@isska.ch

Dr. Marco Moretti, Swiss Federal Institute for Forest, Snow and Landscape Research (WSL), Research Group Insubric Ecosystems, Via Belsoggiorno 22, CH-6500 Bellinzona and Centro protezione chirotteri Ticino, CH-6714 Semione, Switzerland.

e-mail: marco.moretti@wsl.ch

Prof. Dr. Willy Tinner, Institute of Plant Sciences (IPS), University of Bern, Altenbergrain 21, CH-3013 Berne and Paleoecology and Vegetation Dynamics, Institute of Terrestrial Ecosystems (ITES), ETH-Zürich, CH8092 Zurich, Switzerland.

e-mail: willy.tinner@ips.unibe.ch

Manuskripteingang/received/manuscrit entré le 5.5.2008

Annahme zum Druck/accepted for publication/accepté pour limpression: 4.9 .2008 